\title{
Investigation of the Reflectivity Spectrum of the a-Plane Oriented ZnO Epilayers Grown by Plasma-Assisted Molecular Beam Epitaxy from the Gaussian Distribution
}

\author{
Alioune Aidara Diouf ${ }^{1,2, ~ *, ~ B a s s i r o u ~ L o ', ~ A b e l ~ S a m b o u ~}{ }^{1}$, Oumar Sakho', Aboubaker Chedikh Beye ${ }^{1}$ \\ ${ }^{1}$ Faculty of Sciences \& Techniques, Cheikh Anta Diop University, Dakar, Senegal \\ ${ }^{2}$ Department of Nanoscience \& Nanotechnology Research, Dakar American University of Science \& Technology, Somone, Senegal
}

Email address:

aliouneaidara.diouf@ucad.edu.sn (A. A. Diouf)

*Corresponding author

\section{To cite this article:}

Alioune Aidara Diouf, Bassirou Lo, Abel Sambou, Oumar Sakho, Aboubaker Chedikh Beye. Investigation of the Reflectivity Spectrum of the a-Plane Oriented ZnO Epilayers Grown by Plasma-Assisted Molecular Beam Epitaxy from the Gaussian Distribution. American Journal of Optics and Photonics. Vol. 5, No. 5, 2017, pp. 50-54. doi: 10.11648/j.ajop.20170505.11

Received: October 25, 2017; Accepted: November 3, 2017; Published: December 18, 2017

\begin{abstract}
The Photoluminescence spectra at low temperature of the a-plane oriented $\mathrm{ZnO}$ grown on r-plane (011-2) sapphire substrates by plasma-assisted molecular beam epitaxy, showed experimentally three types of excitons A, B and C. In the reflectivity spectra, authors used a program based on the theory of the spatial resonance dispersion Hopfield model to fit the free excitons. The A and B free excitons were fitted together and the C exciton with the band gap. But these fits were not perfect in the transparency zone at low energy. This is mainly due to the fact that the A and B free excitons are closer and the $\mathrm{C}$ exciton is closer to the band gap but another reason is the value of the oscillator strength. In the present work, we present a method taking account the Gaussian distribution, to fit perfectly the excitons A, B and C using almost the same physical parameters than the theory of the spatial resonance dispersion Hopfield model.
\end{abstract}

Keywords: Exciton A, B and C, Gaussian Distribution, Reflectivity Spectrum, a-Plane Oriented ZnO

\section{Introduction}

During the last decade, the zinc oxide $\mathrm{ZnO}$ as thin films, nanowires, nano-spheres as well as the correlative quantum structures, have attracted considerable attention both in theories and experiments. $\mathrm{ZnO}$ presents a particular interest for the researches for news applications because of its wide band gap $\left(\mathrm{E}_{\mathrm{g}}=3.37 \mathrm{eV}\right)$ at room temperature and its larger exciton binding energy $(60 \mathrm{meV})$. Several authors worked on the properties and applications of the semiconductor of $\mathrm{ZnO}$. In solar energy, C. Y. Jiang and al [1] worked on the improvement of the dye-sensitized solar cells with a $\mathrm{ZnO}$ nanoflower photoanode. Yun-Ju Lee and al [2] used $\mathrm{ZnO}$ nanostructures as efficient antireflection layers in solar and others authors used the nanostructures and thin films of $\mathrm{ZnO}$ to study the interest of zinc oxide [3-6]. In the optical properties, B. Lo and al [7] investigated the optical spectroscopy of a-plane oriented $\mathrm{ZnO}$ epilayers grown by plasma-assisted molecular beam epitaxy. In the same way M. Wraback and al [8] as B. Lo and al [7] grew on the r-plane sapphire substrates a $\mathrm{ZnO}$ films and investigated the high contrast, ultrafast optically addressed ultraviolet light modulator based upon optical anisotropy in $\mathrm{ZnO}$ films. E. V. Lavrov and al [9] worked on $\mathrm{ZnO}$ to understand the nature of hydrogen-related shallow donors in $\mathrm{ZnO}$ and J. R. Schneck and al [10] for their applications worked on the polar face dependence of the ultrafast $\mathrm{U}$. V reflectivity of $\mathrm{ZnO}$ single crystal. Hisashi Yoshikawa and al. [11] gave a precious data to the researchers investigating exclusively the optical constants of $\mathrm{ZnO}$. All these works show the important roles which play the zinc oxide in the new technology reason why the authors continued to study it for more information and applications [12-30]. Beside the experimental methods, certain authors used models to investigate the physical 
properties of the zinc oxide or others semiconductors. J. Lagois and al. [21] used the model of the harmonic oscillators and the model oscillators having spatial dispersion to describe the optical dielectrics functions. J. J. Hopfield $[31,32]$ investigated the mixed states of excitons and photons by using the quantum theory of a classical dielectric. B. Lo and al [7] used the Varshni empirical equation to fit the temperature dependent PL of the A free exciton peak energy measured in the case of the perpendicular polarization $(\mathrm{E} \perp \mathrm{c})$ and D. W. Hamby used the Manoogian and Wooley model [33] for the temperature dependent exciton PL of bulk $\mathrm{ZnO}$.

Our aim in this article is to model the optical reflectivity of the a-plane oriented $\mathrm{ZnO}$. We will use the Gaussian distribution in the framework of the "bracketing" method [34-40], to fit the exciton C, which presented a difficulty in certain range of energy because of the oscillator strength related in the ref. [7]. We will compare our results with those obtained in ref. [7, 41-43] using other theoretical treatments. In Section 2, Model, we explained the distribution use which will allow us to determine the theoretical reflectivity of the aplane oriented $\mathrm{ZnO}$ and the results obtained are well discussed in the section 3 .

\section{Model}

To model the optical properties we used the Gaussian distribution defined by:

$$
R(\omega)=R_{0}+\alpha \cdot \sum_{i=1}^{n}\left(\frac{A_{i}}{\Gamma_{i} \sqrt{\frac{\pi}{2}}}\right) \times \exp -\left(\frac{h^{2}\left(\omega-\omega_{0_{i}}\right)^{2}}{2 \pi^{2} \Gamma_{i}^{2}}\right)
$$

Where

$$
\begin{aligned}
& \alpha=\frac{4 \pi N e_{0}^{2}}{m^{*}} \text { With } e_{0}=e / 4 \pi \varepsilon_{0} \text { and } m^{*}=0.59 m_{0} \\
& A_{i}=\int_{\omega_{1}}^{\omega_{n}} \frac{\delta r(\omega)}{\delta \omega} d \omega=\int_{\omega_{1}}^{\omega_{2}} \frac{\delta r_{1}(\omega)}{\delta \omega} d \omega+\int_{\omega_{2}}^{\omega_{3}} \frac{\delta r_{2}(\omega)}{\delta \omega} d \omega+\cdots+\int_{\omega_{n-1}}^{\omega_{n}} \frac{\delta r_{n}(\omega)}{\delta \omega} d \omega
\end{aligned}
$$

and

$$
r(\omega)=\left(\frac{1}{\Gamma \sqrt{\frac{\pi}{2}}}\right) \times \exp -\left(\frac{h^{2}\left(\omega-\omega_{0}\right)^{2}}{2 \pi^{2} \Gamma^{2}}\right)
$$

$\mathrm{R}_{0}$ : Value of the reflectivity for $\omega=0$

A: Spectral area

$\Gamma$ : Spectral Widening

$\omega_{0}$ : Resonance frequency

$\mathrm{n}$ : Number of intervals

$\alpha$ : Oscillator strength

$\mathrm{N}$ : Number of particles

$\mathrm{e}_{0}$ : Elementary charge electron

$\mathrm{m}^{*}$ : Effective masse.

To fit the excitons A, B and C, we started to compare two distributions Lorentzian and Gaussian (cf. Figure 1, Figure 3 Vs Figure 2, Figure 4). We noted that in the range of energy between $3.375 \mathrm{eV}$ and $3.425 \mathrm{eV}$, which represent the reflectivity pic energy range, both distributions are accurate with the experimental reflectivity curves. But from $3.275 \mathrm{eV}$ to $3.375 \mathrm{eV}$ representing the range of transparency zone, we observed that the Gaussian distribution was more accurate than the Lorentzian distribution. We can explained this latter by the fact that, because of the frequencies, the Lorentzian distribution does not take account certain phenomenon when the frequencies $(\omega)$ are lower than the resonance frequency $\left(\omega_{0}\right)$. The comparison $\omega<<\omega_{0}$ represents the range of transparence, a reason why in Figure 3 and Figure 4 we did not observe an accuracy between the experimental and theoretical reflectivity curves by the Lorentzian distribution for the excitons $\mathrm{A}, \mathrm{B}$ and $\mathrm{C}$ in this range of frequency. Elsewhere, others authors already used [42] the Gaussian distribution to fit the emission spectra of the A-band $\mathrm{ZnO}$, which related to the presence of acceptor impurities through different radiative mechanisms like donor-acceptor pair, a free bound transition or an exciton bound to a defect. All these facts and observations allowed us to work, in this paper, with a Gaussian instead of a Lorentzian distribution.

\section{Results and Discussion}

The reflectivity spectrum informs more about the free excitons peaks positions than the photoluminescence. Because of the pre-eminence of the bound excitons B. Lo and al. [7] observed in their experiments of reflectivity, in the case where $E \perp c$ polarization, two free excitons called $\mathrm{A}$ and $\mathrm{B}$ at energy positions $\mathrm{E}_{\mathrm{A}}=3.398 \mathrm{eV}$ and $\mathrm{E}_{\mathrm{B}}=3.410 \mathrm{eV}$ where their equivalences in frequencies are respectively $\omega_{\mathrm{A}}=$ $0.516 \times 10^{14} \mathrm{~s}^{-1}$ and $\omega_{\mathrm{B}}=0.518 \times 10^{14} \mathrm{~s}^{-1}$. Authors [7] used the theory of the spatial resonance dispersion Hopfield model [32] with the relative dielectric constant defined by:

$$
\varepsilon_{r}=1+\frac{4 \pi N e_{0}^{2}}{m^{*}}\left[\frac{\omega_{0}^{2}-\omega^{2}}{\left(\omega_{0}^{2}-\omega^{2}\right)^{2}-\Gamma^{2} \omega^{2}}\right]
$$

and

$$
R=\frac{(n-1)^{2}+k_{0}}{(n+1)^{2}+k_{0}}
$$

to fit the free excitons A, B (cf. Figure 1). Where the coefficient $4 \pi N e_{0}^{2} / m *$ is related to the oscillator strength $(\alpha)$, " $\omega_{0} "$ is the resonance frequency, $\mathrm{e}_{0}=\mathrm{e} / 4 \pi \varepsilon_{0}$ with "e" designated as the elementary charge electron, " $R$ " the reflection coefficient, " $\mathrm{n}$ " the index of the medium and " $\mathrm{k}_{0}$ " the extinction coefficient. In the present study, the fact that we observe in the reflectivity a succession of curves which have the behavior of a Gaussian, allowed us to make a program using the Gaussian distribution. This latter, applied to the "bracketing" method [36] allowed us to model the reflectivity curves found experimentally by authors [7]. The results obtained by simulation are summarized in the table 1 
(cf. Appendix) compared to the results found by the authors [7] (table 2, cf. Appendix). A comparison of the tables show that the Gaussian distribution (c. f. Figure 1 and Figure 2) is more accurate than the model used by the authors $[7,41]$ to fit the exciton A, B. We insist on the fact that the parameters summarized in the table 1 are used to model the theoretical reflectivity of the exciton $\mathrm{A}, \mathrm{B}$. In table 2 , these parameters have been used by the authors [7] to have their best fit of the free excitons. Our method, with the Gaussian distribution, allowed us, furthermore the excitons A, B (cf. Figure 1), to fit the free exciton $\mathrm{C}$ shown in the Figure 2. The parameters used to fit the free exciton $\mathrm{C}$ are summarized in Table 1 (cf. Appendix). Unless the others models [41, 45, 46, 47, 48], which are limited on the investigation of the c-plane oriented $\mathrm{ZnO}$, our method with the Gaussian distribution, moreover cplane oriented $\mathrm{ZnO}$, allowed us to investigate the structure of the a-plane oriented $\mathrm{ZnO}$ and we fitted in the same time the excitons $\mathrm{A}, \mathrm{B}$ and $\mathrm{C}$ something which we did not see in the literature with the others models.

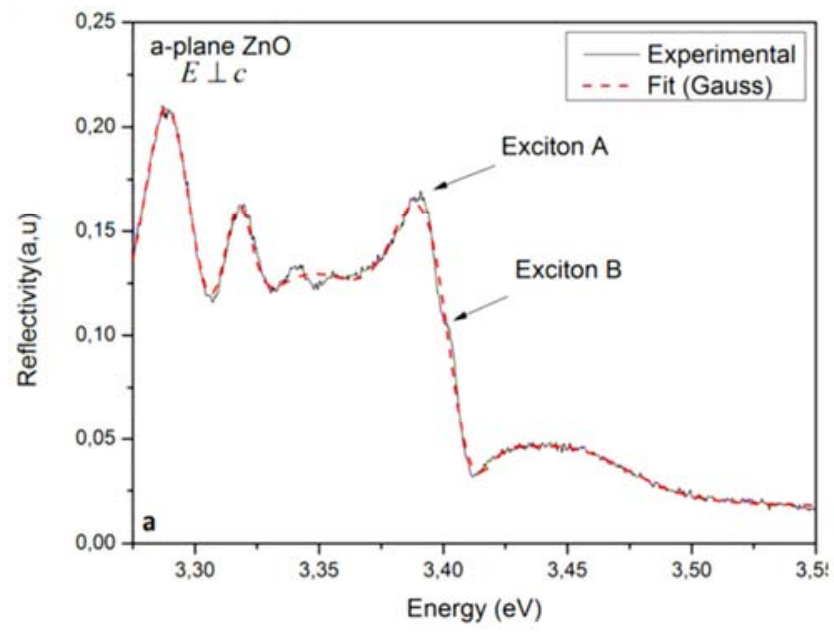

Figure 1. Experimental and Theoretical (Gaussian distribution) reflectivity of exciton $A$ and $B$.

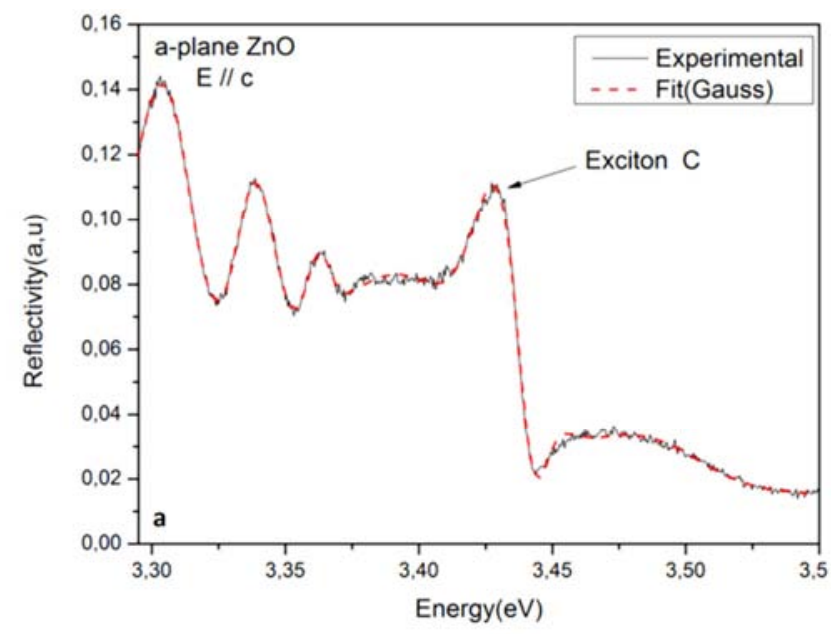

Figure 2. Experimental and Theoretical (Gaussian distribution) reflectivity of exciton $C$.

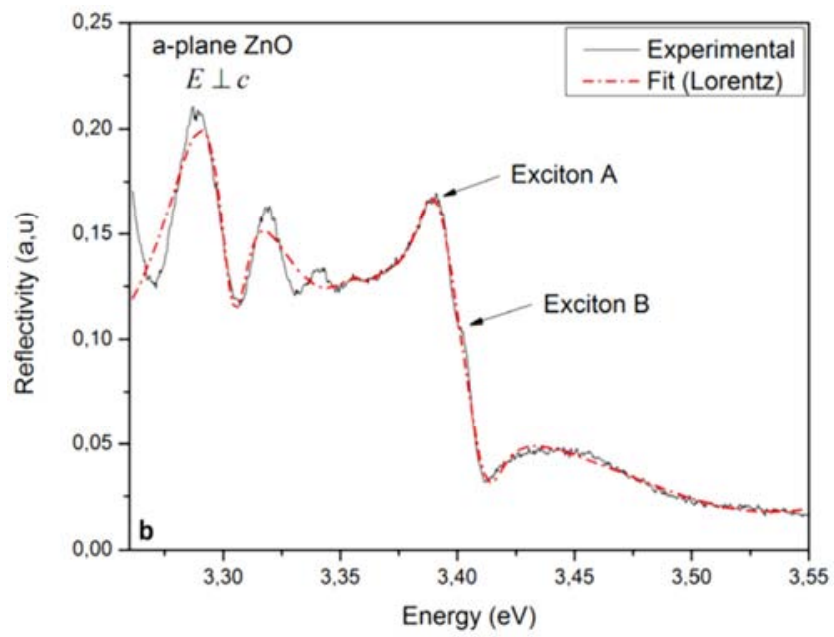

Figure 3. Experimental and Theoretical (Lorentzian distribution) reflectivity of exciton $A$ and $B$.

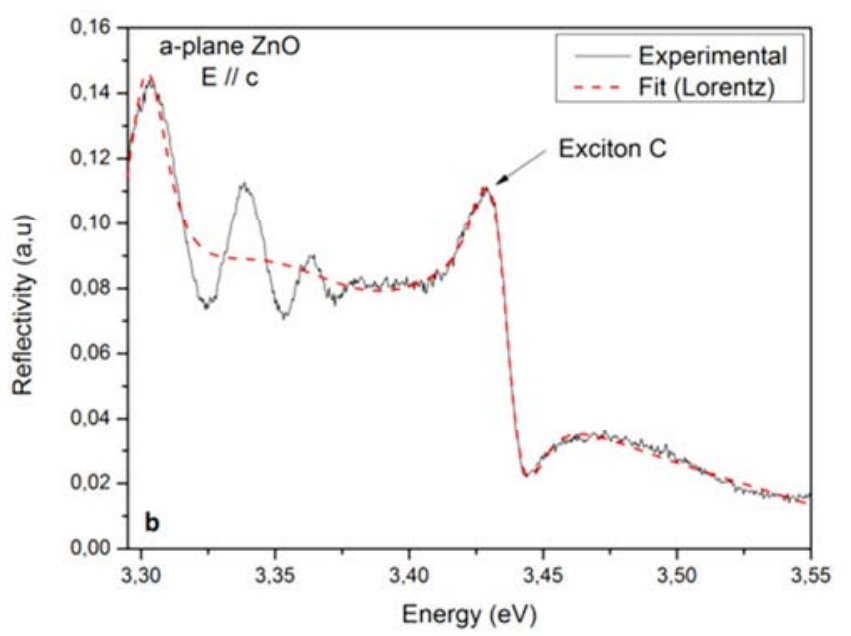

Figure 4. Experimental and Theoretical (Lorentzian distribution) reflectivity of exciton $C$.

\section{Conclusion}

In summary, we investigated the reflectivity of a-plane oriented $\mathrm{ZnO}$ thin film. Our model based on the Gaussian distribution allowed us to fit the free excitons $\mathrm{A}, \mathrm{B}$ and $\mathrm{C}$ in the range of reflectivity pic energy and even in the transparency zone. We compared our model with those used by the authors $[41,45,46,47,48]$ and we validated our model with the experimental results in the ref [7]. The interest of the obtained results is that they can be applied to semi-conductors which have the same structural properties such as $\mathrm{GaN}, \mathrm{CdS}, \alpha-\mathrm{SiC}$ etc. to model and investigate the optical properties and even other physical properties [49]. Our next perspective is to investigate the diluted magnetic system type $\mathrm{ZnO}$ for the understanding of their magnetics [50] properties. 


\section{Acknowledgements}

Dr. Bassirou Lo gratefully acknowledges about your help for the experimental results for the investigation of the a- plane oriented ZnO. Pr. Beye gratefully acknowledges for your hospitality in your Nanoscience Laboratory at the University Cheikh Anta Diop of Dakar where i performed these results.

\section{Appendix}

Table 1. Values of $A, B$ and $C$ free exciton frequency $(\omega)\left(s^{-1}\right)$, the spatial widening $(\Gamma)$, the effective mass ( $\left.m^{*}\right)$ and the Area $\left(A_{i}\right)$ of the reflective curves obtained by using the Gaussian distribution.

\begin{tabular}{|c|c|c|c|c|}
\hline Free Exciton & Frequency $\left(\omega_{0}\right)\left(\mathrm{s}^{-1}\right)$ & Spectral widening $(\Gamma)\left(\mathrm{m} \mathrm{s}^{-1}\right)$ & Effective mass (m*) & Area \\
\hline A & $0.515 \mathrm{E} 14(\mathrm{E}=3.389 \mathrm{eV})$ & $3.251 \mathrm{E} 12(\Gamma=2.14 \mathrm{meV})$ & $0.59 \mathrm{~m}_{0}$ & $2.2 \mathrm{E}-4$ \\
\hline B & $0.516 \mathrm{E} 14(\mathrm{E}=3.401 \mathrm{eV})$ & $3.996 \mathrm{E} 14(\Gamma=26.3 \mathrm{meV})$ & $0.59 \mathrm{~m}_{0}$ & $1.12 \mathrm{E}-4$ \\
\hline $\mathrm{C}$ & $0.521 \mathrm{E} 14(\mathrm{E}=3.430 \mathrm{eV})$ & $2.53 \mathrm{E} 10(\Gamma=1.66 \mathrm{meV})$ & $0.59 \mathrm{~m}_{0}$ & $1.3 \mathrm{E}-4$ \\
\hline
\end{tabular}

Table 2. Values of $A$ and $B$ free exciton Energy $E(\mathrm{eV})$, Oscillator Strength $(\alpha)$, the spatial widening ( $\Gamma$ ) and the effective mass ( $m *$ ) of the exciton obtained by using the Hopfield model [7].

\begin{tabular}{lllll}
\hline Free Exciton & Energy $(\mathbf{E})(\mathbf{e V})$ & Oscillator Strength $(\boldsymbol{\alpha})$ & Spectral widening $(\boldsymbol{\Gamma})(\mathbf{m e V})$ & $\mathbf{E f f e c t i v e ~} \mathbf{m a s s}(\mathbf{m} *)$ \\
\hline A & 3.393 & 1.708 & 10.38 & $0.59 \mathrm{~m}_{0}$ \\
B & 3.403 & 0.77 & 11.479 & $0.59 \mathrm{~m}_{0}$ \\
\hline
\end{tabular}

\section{References}

[1] C. Y. Jiang, X. W. Sun, G. Q. Lo, D. L. Kwong, J. X. Wang, applied physics letters 90, 263501 (2007).

[2] Yun-Ju Lee, Douglas S. Ruby, David W. Peters, Bonnie B. Mckenzie, Julia W. P. Hsu, Nano Letters 2008, Vol.8, N5, 1501-1505.

[3] C. Eisele, C. E. Nebel, M. Stutzmann, j. appl. Phys. 89, 7722 (2001).

[4] J. Springer, B. Reeh, W. Reetz, J. Müller, M. Vanecek, solar energy materials \& solar cells 85 (2005) 1- 11.

[5] Takashi Fujibayashi, Tkuya Matsui, Michio Kondo, applied physics letters 88,18350 (2006).

[6] Kannan Ramanathan, Miguel A. Contreras, Craig L. Perkins, Sally Asher, Falash S. Hasoon, James Keane, David Young, Manuel Romero, Wyatt Metzger, Rommel Noufi, James Ward, Anna Duda, Prog. Photovolt: res. Appl 2003; 11: 225-230.

[7] B. Lo, M. B. Gaye, A. Dioum, C. M. Mohrain, M. S. Tall, J. M. Chauveau, M. Doninelli Tesseire, S. Ndiaye, A. C. Beye, Appl. Phys. A (2014) 115: 257-261.

[8] M. Wraback, H. Shen, S. Liang, C. R. Gorla, and Y. Lu, Applied Physics Letters 74, 507 (1999).

[9] E. V. Lavrov, F. Börrnert, J. Weber, Physica B 376-377 (2006) 694-698.

[10] J. R. Schneck, E. Belloti, P. Lammarre, L. D. Ziegler, Applied Physics letters 93, 102-111 (2008).

[11] Hisashi Yoshikawa, Sadao Adachi, Jpn. Appl. Phys. Vol.36 (1997) pp. 6237-6243.

[12] S. Bloom, I. Ortenburger, phys. Stat. sol. 58, 561 (1973).

[13] Y. C. Liu, S. K. Tung, J. H. Hsieh, Journal of crystal growth 287 (2006) 105-111.

[14] S. F. Yu, Clement, Yuen, S. P. Lau, W. I. Park, Gyu-Chui Yi, Applied Physics Letters 84,3241 (2004).
[15] J. M. Jensen, A. B. Oelkers, R. Toivola, D. C. Johnson, Chem. Mater.2002, 14, 2276-2282.

[16] Zuowan Zhou, Longsheng Chu, Shuchun Hu, Materials Science and Engineering B 126 (2006) 93-96.

[17] K. Hümmer, phys. Stat. sol. 56 (b), 249 (1973).

[18] Claude Lévy-Clément, ramon tena-Zaera, margaret A. Ryan, Abou Katty, Gary Hodes, adv. Mater 2005, 17, 1512-1515.

[19] F. Hamdani, A. Botchkarev, W. Kim, H. Morkoço, M. Yeadon, J. M. Gibson, S. C. Y. Tsen, David J. Smith, D. C. Reynolds, D. C. Look, K. Evans, C. W. Litton, W. C. Michel, P. Hemenger, Applied physics letters 70, 467 (1997).

[20] Z. C. Jin, I. Hamberg, C. G. Granqvist, J. Appl. Phys 64, 5117 (1988).

[21] J. Lagois, K. Hümmer, phys. Stat. sol. (b) 72, 393 (1975).

[22] M. Kunat, St. Gil Girol, Th. Becker, U. Burghaus, Ch. Wöll, Physical Review B 66, 081402® 2002.

[23] Xiaodong Yang, Jingwen Zhang, Zhen Bi, Yongning He, Qing'an $\mathrm{Xu}$, Hongbo Wang, Weifeng Zhang, Xun Hou, Journal of Crystal Growth 284 (2005) 123-128.

[24] R. J. Collins, D. A. Kleinman, J. Phys. Chem. Solids Pergamon Press 1959, vol. 11, pp. 190-194.

[25] R. L. Hengehold, R. J. Almassy, Physical Review B Vol. 1, N ${ }^{\circ}$ 12 (1970).

[26] Sheng Chu, Mario Olmedo, Zheng Yang, Jieying Kong, Jianlin Liu, Applied Physics Letters 93, 181106 (2008).

[27] M. Lorentz, M. Hochmuch, R. Schmidt-Grund, E. M. Kaidashevi, M. Grundmann, Ann. Phys. (Leipzig) 13, $\mathrm{N}^{\circ} 1$, 59-60 (2004).

[28] R. Klucker, H. Nelkowski, Y. S. Park, M. Skibowski, T. S. Wagner, Phys. Stat. sol. 45, 265 (1971).

[29] Run Wu, Changsheng Xie, Materials Research Bulletin (39 (20014) 637-645.

[30] E. F. Venger, A. V. Melnichuk, L. Yu. Melnichuck, A. Pasechnik, phys. Stat. sol. (b) 188, 823 (1995). 
[31] J. J. Hopfield, physical review, vol. 112, 5, 1555-1567 (1958).

[32] J. J. Hopfield, D. G. Thomas, physical review, vol. 132,2,563$572(1963)$

[33] D. W. Hamby, D. A. Lucca, M. J. Klopfstein, G. Cantwell, Journal of Applied Physics 93,3214 (2003).

[34] A. Björk, Numerical Methods for last Squares Problems, SIAM, Philadelphia, PA.

[35] A. L. Dulmage, N. S. Mendelsohn, Journal of the Society for Industrial and Applied Mathematics, 11 (1), 183-194 (1963).

[36] Manfred Gilli, Méthodes Numériques, pp. 1-132 (2006).

[37] M. Gilli, M. Garbely, Journal of Economic Dynamics and Control 20, 1541-1556 (1996).

[38] G. H. Golub, C. F. Van Loan, J. Numer. Anal., 17 (6), 883-893 (1980).

[39] C. L. Lawson, R. J. Hanson, D. R. Kincaid, F. T. Krogh, ACM Transactions on Mathematical Software, Vol 5, N³, 308-323 (1979).

[40] W. H. Press, B. P. Flannery, S. A. Teukolsky, W. T. Vetterling, Geological Magazine 127, 376-377 (1990).

[41] T. Makino, Y. Segawa, M. Kawasaki, A. Ohtomo, Journal of Crystal Growth 287 (2006) 124-127.

[42] D. Tainoff, B. Masenelli, P. Mélinon, A. Belsky, G. Ledoux,
D. Amans, C. Dujardin, N. Fedorov, P. Martin, Physical Review B 81, 115304 (2010).

[43] D. G. Thomas, J. Phys. Solids Pergamon Press 1960. Vol.15. pp. 86-96.

[44] D. C. Reynolds, D. C. Look, B. Jogai, Physical Review B 60 (4), 2340 (5).

[45] S. I. Pekar, J. Exptl. Theoret. Phys. (U. S. S. R) 34, 1176-1188 (1958).

[46] S. I. Pekar, J. Exptl. Theoret. Phys. (U. S. S. R) 38, 17861797, (1960).

[47] J. Frenkel, Physical Review, Vol 54, pp.17-44 (1930).

[48] Gregory H. Wannier, Physical Review, Vol 37, pp.191-197 (1937).

[49] Alioune Aidara Diouf, Bassirou Lo, Famara Dieme, Abdourahmane Mbodji, Aboubaker Chedikh Beye, Computer Simulation of the Dielectrics Properties in the Dense Circles, Colloid and Surface Science. Vol. 2, No. 3, 2017, pp. 107-113. doi: 10.11648/j.css.20170203.13

[50] Alioune Aidara Diouf, Bassirou Lo, Alhadj Hisseine Issaka Ali, Aboubaker Chedikh Beye. Comparative Investigation and Generalized of the Core/Shell Effects on the Magnetics Properties in the Ferromagnetic Cubic Nanoparticles by the Transverse Ising Model. American Journal of Nanomaterials. Vol. 4, No. 1, 2016, pp 1-7. http://pubs.sciepub.com/ajn/4/1/1. 